\title{
FACTORES PSICOSOCIALES Y CULTURALES QUE INFLUYEN EN EL EVENTO DE LA MENARQUIA EN ADOLESCENTES POSMENÁRQUICAS
}

\author{
Electra González A. ${ }^{1 a}$, Adela Montero V. ${ }^{1}$ \\ 1 Centro de Medicina Reproductiva y Desarrollo Integral de la Adolescencia (CEMERA), Facultad de Medicina, \\ Universidad de Chile.
}

a Asistente Social.

\section{RESUMEN}

Introducción: La menarquia es un evento de vida significativo con importantes implicaciones culturales, sociales y personales. Objetivo: Conocer y entender mejor el contexto psicosocial en que ocurre la menarquia considerando la edad de ocurrencia, fuentes y calidad de la información o preparación recibida, y las reacciones emocionales experimentadas. Método: Se entrevistaron adolescentes entre 11 y 19 años que consultaron en CEMERA entre 2005 y 2006. Se aplicó un cuestionario especialmente diseñado y se aseguró confidencialidad y anonimato de sus respuestas. Resultados: Participaron 826 adolescentes. $34,6 \%$ de ellas tuvieron la menarquia entre los 9 y 11 años, 53,1\% entre 12 y 13 años y $12,3 \%$ entre los 14 y 16 años. $88,6 \%$ recibieron información sobre el tema previo a la menarquia. $81,3 \%$ de las madres fueron quienes informaron a sus hijas, seguidas por familiares mujeres en $6,8 \%$, colegio en $4,6 \% ; 78 \%$ de las adolescentes que recibieron información indicaron no sentirse preparadas al momento del evento. $23,6 \%$ reportó sentirse muy mal, $21 \%$ asustada, $20,5 \%$ extraña, $4 \%$ indiferente y $30,8 \%$ indicó sentirse feliz con la menarquia. Conclusión: Si bien en la actualidad las niñas tienen acceso a una serie de fuentes de información acerca de la menstruación es principalmente la madre, el colegio y los profesionales de salud quienes juegan un rol primordial en su preparación en este aspecto. Pero esta información tiende a estar focalizada solo en aspectos higiénicos y biológicos inmediatos y obvios pero desconectados de la experiencia del propio cuerpo de las niñas como de su emocionalidad.

\section{PALABRAS CLAVE: Menarquia, fuentes de información, factores socioculturales}

\section{SUMMARY}

Background: Menarche is a significant life event with important cultural, social and personal implications. Objectives: To know and to understand in a better way, the psychosocial context in which menarche takes place, considering age occurrence, sources and quality of information, training received and the emotional reactions experienced. Methods: In 2005 and 2006, adolescents from 12 to 19 years old, who consulted at an Adolescents Clinic, took a specially designed questionnaire and they were guaranteed confidentiality and anonymity in their answers. Results: 826 adolescents took part in this study; $34.6 \%$ of them had the menarche between $9-12$ years old, $53.1 \%$ between $12-13$ years old and $12.3 \%$ between $12-14$ years old; $88.6 \%$ got information previously to menarche; $81.3 \%$ mothers were who gave the corresponding information to their daughters, followed by their female relatives in a $6.8 \%$, school in a $4.6 \% ; 78 \%$ of the total number 
of adolescents who got adequate information, pointed out that they did not feel prepared enough at the moment of the event; $23.6 \%$ reported they felt very bad, $21 \%$ were frightened, $20.5 \%$ awkward, $4 \%$ unconcerned and only $30.8 \%$ reported they felt happy when they experienced menarche. Conclusion: Although, nowadays girls have a major access to a various sources of information about menarche, it is mainly mother, school and health professionals who participate in their training on this area. But this information tends to be focused on hygienically and biological aspects that are immediate and obvious, but it is disconnected from the experience of the girl bodies as of their emotionality.

\section{KEY WORDS: Menarche, information source, sociocultural factors}

\section{INTRODUCCIÓN}

La pubertad esta marcada por la adquisición de la capacidad reproductiva y por el desarrollo de los caracteres secundarios. Estos cambios que llevan a la maduración sexual dura entre 4 a 5 años. En las niñas, el indicador fisiológico de la pubertad es la primera menstruación, conocida como menarquia. La menarquia es un evento de vida significativo con importantes implicaciones culturales, sociales y personales. La partida de la menstruación es un evento marcador en la vida de las mujeres y una fecha que ellas nunca olvidarán, Sin embargo, los reportes, a menudo retrospectivos, acerca de la edad de ocurrencia y factores que rodean el evento tienen un gran sesgo de la memoria y la distorsión aumenta en la medida en que aumenta los años desde la ocurrencia de la primera menstruación $(1,2)$. En relación a la edad a la menarquia los estudios muestran que ésta ha declinado considerablemente en los dos últimos siglos, siendo 16 años a mediados del siglo 18. Actualmente, el promedio de edad a la menarquia es 12,8 años para la mayoría de las mujeres, con rangos que van desde 10,5 a 15 años, con algunas diferencias raciales. Las implicaciones psicosociales asociadas a la diferencias de edad de la menarquia pueden ser de mayor consecuencia para las niñas que la menarquia en si misma (1-3).

Independiente de la edad en que ocurre, la menarquia representa un símbolo concreto de pasaje de niña a mujer; y así es reconocido en muchas culturas alrededor del mundo, incluso algunas tradiciones marcan este evento con regalos de artículos de color rojo o comidas simbólicas especiales (4). A diferencia de otros cambios puberales graduales como desarrollo de mamas, crecimiento del vello púbico, la menarquia ocurre de manera más bien súbita y sin un predictor preciso.

Estudios transculturales de las reacciones emocionales de las mujeres ante el evento han tenido diversos resultados. Mientras que algunas mujeres recuerdan haberse sentido más maduras, más grandes, orgullosas y felices, otras reportan haber tenido experiencias negativas, tales como estar asustada, preocupada, ansiosa, de mal humor y avergonzada con el inicio de la menstruación. Algunos autores sugieren que pudiera haber consecuencias psicológicas adversas para las adolescentes cuando no están preparadas ni informadas acerca de la menarquia (3-5).

A pesar que la gente a través de las culturas ha llegado a ser bastante más franca y abierta en sus actitudes hacia varios temas sexuales, aun es incómodo conversar acerca de la menstruación y se mira más bien como un tabú. Algunos estudios muestran que las actitudes de la gente hacia la menstruación son generalmente negativas y a menudo asocian la menstruación con malestar físico, aumento de la emocionalidad y la disrupción de actividades e interacciones sociales (6-7).

Hay autores que sostienen que las mujeres que perciben la menstruación como debilitante y disruptiva tienen mayor probabilidad de experimentar más síntomas relacionados con la menstruación, tales como dolor menstrual y efectos negativos. La sintomatología depresiva también aumenta. Estos hallazgos indican lo significativo que son las actitudes que afectan las experiencias de la menarquia y menstruación de las mujeres (7-10). Por otro lado, la menarquia marca el comienzo de la vida menstrual y reproductiva de las mujeres y ocurre durante la adolescencia temprana, justamente cuando las niñas están empezando a separarse emocionalmente de sus familias, tan bien como a manejar su sexualidad femenina $(11,12)$.

Los antropólogos han documentado una variación transcultural en los comportamientos menstruales, indicando así, que mucho de lo que se ha observado puede ser atribuido a expectativas sociales más bien que a mandatos biológicos. En cualquier sociedad, el significado de la menstruación para la niña y su interpretación personal de su función puede estar relacionado a sus imágenes del cuerpo general y genital, y esto puede tener importantes consecuencias en términos de su sexualidad y elección anticonceptiva futura. Los investigadores preocupa- 
dos con la sexualidad adolescente y con el embarazo se han interesado también en la socialización menstrual (11-16).

A pesar de la atención creciente que ha sido dada a los componentes sociales de la experiencia menstrual, el proceso de transmisión de la menstruación en el tiempo crítico de la menarquia ha sido poco estudiado. Los psiquiatras preocupados con el desarrollo adolescente han ofrecido observación acerca del significado de la menarquia, pero el hecho es que estas están basadas en población de pacientes lo cual no permite generalizar. Otros estudios se han focalizado en mujeres más jóvenes de población no siquiátricas, pero los pequeños números de niñas premenárquicas incluidas hace también difícil generalizar. Otros estudios han recopilados datos retrospectivos de mujeres mayores a quienes se les ha pedido que recuerden eventos ocurridos alrededor de su primera menstruación. Las respuestas en tales estudios pueden ser opacadas por los años en que ocurrió el evento. Sin embargo, ciertos temas relacionados con la menarquia pueden aportar información sobre las reacciones mirando la primera menstruación en niñas postmenárquicas (17-23).

Si bien las niñas tienen acceso a una variedad de fuentes de información acerca de la menstruación son principalmente los padres, los profesionales de salud y el colegio quienes juegan un rol primordial en su preparación en este aspecto. Pero esta información tiende a estar focalizada en aspectos higiénicos y biológicos inmediatos y obvios pero desconectados de la experiencia del propio cuerpo de las niñas. En esta edad las niñas carecen de familiaridad con las partes del cuerpo conectadas con el ciclo menstrual, y en particular con los órganos reproductivos. Sin duda, educar a las mujeres para la vida menstrual es compleja y multidimensional pero pueden distinguirse tres aspectos importantes que deberían ser considerados en esta preparación, entregar conocimiento acerca de la biología de la menstruación y la higiene menstrual, apoyo emocional y tranquilidad y el significado psicosocial.

El objetivo de este estudio fue conocer y entender mejor el contexto psicosocial en que ocurre la menarquia considerando la edad a la menarquia, fuentes y calidad de la información, preparación recibida y las reacciones emocionales al evento en adolescentes postmenárquicas.

\section{MATERIAL Y MÉTODO}

Diseño de estudio transversal y analítico. El universo de estudio estuvo constituido por adolescen- tes mujeres entre 11 y 19 años que consultaron en el Centro de Medicina Reproductiva y Desarrollo Integral de la Adolescencia (CEMERA) entre los años 2005 y 2006. Una entrevista estructurada diseñada como instrumento de recolección para la investigación se les aplicó en la primera consulta. Las adolescentes firmaron un consentimiento informado y se les aseguro la confidencialidad y anonimato de sus respuestas. Fueron incluidas en el estudio solamente las adolescentes que ya habían tenido su menarquia. La entrevista incluyó variables como edad a la menarquia, fuentes de información, preparación, respuesta emocional a la menarquia, rol de los padres en la preparación. Incluyó además datos demográficos como edad a la entrevista, escolaridad y variables familiares como relación con padres, comunicación familiar, escolaridad parental.

Los resultados para las variables medidas en escala nominal fueron expresados como porcentajes. Se realizaron análisis uni y bivariado, aplicándose prueba de Chi2, utilizando el software EPIINFO 6.0.

\section{RESULTADOS}

Los resultados corresponden a 826 adolescentes entre 11 y 19 años postmenárquicas. El 67,5\% tenía entre 16 y 17 años al momento de la entrevista. Un $57,2 \%$ cursaba entre $3^{\circ}$ y $4^{\circ}$ medio. Las adolescentes provenían de la mayoría de las comunas de la Región Metropolitana, exceptuando Vitacura y Las Condes. El nivel socioeconómico medido por la escala de Graffar modificada fue medio-bajo.

La edad promedio a la menarquia de toda la población estudiada fue 12,02 años (rango: 8 - 16 años). El 34,6\% de las niñas tuvieron su menarquia antes de los 12 años, 53,1\% entre 12 y 13 años y $12,3 \%$ a los 14 años y más.

El $88,6 \%$ (695) de las adolescentes respondieron que habían recibido información previo a la menarquia. La principal fuente de información fueron las madres en un $81,3 \%, 6,8 \%$ otro familiar, el colegio en $4,6 \%$ e incluía al padre solo el $4,3 \%$. El $78,1 \%$ reportó que la información recibida fue suficiente y adecuada.

El $21,1 \%$ se asustó al momento de la menarquia, $23,6 \%$ se sintió mal, $20,5 \%$ se sintió extraña, $4,0 \%$ indiferente y $30,8 \%$ refirió sentirse contenta o feliz (Tabla I).Las niñas que tuvieron la menarquia precoz (entre 8 y 11 años), reportaron en mayor proporción que no recibieron información previa y que reaccionaron mal y asustadas al momento de la primera regla, cuando se comparan con aquellas que tuvieron su menarquia entre 12 y 13 años y 
Tabla I

DISTRIBUCIÓN DE LAS ADOLESCENTES SEGÚN CARACTERÍSTICAS PERSONALES

\begin{tabular}{|c|c|c|c|}
\hline Variable & Categorías & $\mathrm{n}$ & $\%$ \\
\hline \multirow[t]{4}{*}{ Edad a la entrevista } & 11 a 13 años & 40 & 4,6 \\
\hline & 14 a 15 años & 199 & 22,8 \\
\hline & 16 a 17 años & 588 & 67,5 \\
\hline & 18 a 19 años & 44 & 5,1 \\
\hline \multirow[t]{4}{*}{ Escolaridad } & $1^{\circ}$ a $8^{\circ}$ básico & 84 & 9,6 \\
\hline & $1^{\circ}$ a $2^{\circ}$ medio & 290 & 33,0 \\
\hline & $3^{\circ}$ a $4^{\circ}$ medio & 502 & 57,2 \\
\hline & Superior & 2 & 0,2 \\
\hline \multirow[t]{3}{*}{ Edad a la menarquia } & 9 a 11 años & 292 & 35,4 \\
\hline & 12 y 13 años & 435 & 52,7 \\
\hline & 14 y 16 años & 99 & 12,0 \\
\hline Recibieron información & $\mathrm{Si}$ & 695 & 88,6 \\
\hline previa a la menarquia & No & 89 & 11,4 \\
\hline Principal fuente de & Madre & 529 & 81,3 \\
\hline \multirow[t]{5}{*}{ información } & Amigas & 13 & 2,0 \\
\hline & Colegio & 30 & 4,6 \\
\hline & Madre y padre & 28 & 4,3 \\
\hline & Familiares & 44 & 6,8 \\
\hline & Otros & 7 & 1,1 \\
\hline Calidad de la información & Suficiente & 410 & 78,1 \\
\hline \multirow[t]{2}{*}{ recibida } & Regular & 38 & 7,2 \\
\hline & Insuficiente & 77 & 14,7 \\
\hline Reacción que experimentó & Se asustó & 162 & 21,1 \\
\hline \multirow[t]{4}{*}{ cuando tuvo su menarquia } & Se sintió mal & 181 & 23,6 \\
\hline & Se sintió extraña & 157 & 20,5 \\
\hline & Indiferente & 31 & 4,0 \\
\hline & Se sintió feliz & 236 & 30,8 \\
\hline
\end{tabular}

entre 14 y 16 años. La calidad de la información recibida y la fuente de información no mostró diferencia por grupos (Tabla II).

Las niñas cuyas madres fueron madres adolescentes reportaron en mayor proporción que se sintieron mal o asustadas al momento de la primera menstruación, comparadas con niñas cuyas madres no fueron madres adolescentes. La calidad de la relación con los padres, comunicación familiar, participación social, religión y con quien conversa sobre sexualidad no mostró diferencia por grupo (Tabla III).

\section{DISCUSIÓN}

A pesar que la menarquia es un evento marcador en la vida de las mujeres y que este evento había ocurrido en un lapso entre 1 a 3 años, en la 
Tabla II

\section{DISTRIBUCIÓN DE VARIABLES SELECCIONADAS DE LAS ADOLESCENTES SEGÚN EDAD A LA MENARQUIA}

\begin{tabular}{|c|c|c|c|c|c|c|c|c|}
\hline \multirow[t]{2}{*}{ Variable } & \multirow[t]{2}{*}{ Categorías } & \multicolumn{2}{|c|}{8 a 11 años } & \multicolumn{2}{|c|}{12 a 13 años } & \multicolumn{2}{|c|}{14 a 16 años } & \multirow[t]{2}{*}{ Valor $\mathrm{p}$} \\
\hline & & $\mathrm{n}$ & $\%$ & $\mathrm{n}$ & $\%$ & $\mathrm{n}$ & $\%$ & \\
\hline Recibió & $\mathrm{Si}$ & 226 & 83,4 & 375 & 90,1 & 93 & 96,9 & 0,00 \\
\hline información & No & 45 & 16,6 & 41 & 9,9 & 3 & 3,1 & \\
\hline Reacción a & Mal & 66 & 24,2 & 90 & 22,3 & 24 & 26,7 & 0,04 \\
\hline la & Asustada & 65 & 23,8 & 84 & 20,8 & 13 & 14,4 & \\
\hline \multirow[t]{3}{*}{ menarquia } & Extraña & 63 & 23,1 & 72 & 17,9 & 22 & 24,4 & \\
\hline & Indiferente & 12 & 4,4 & 19 & 4,7 & 0 & 0 & \\
\hline & Feliz & 67 & 24,5 & 138 & 34,2 & 31 & 34,4 & \\
\hline Calidad de & Adecuada & 134 & 79,3 & 220 & 77,5 & 56 & 77,8 & NS \\
\hline la & Regular & 14 & 8,3 & 17 & 6,0 & 7 & 9,7 & \\
\hline información & No adecuada & 21 & 12,4 & 47 & 16,5 & 9 & 12,5 & \\
\hline Fuente de & Madre & 175 & 83,7 & 280 & 80,0 & 73 & 80,2 & NS \\
\hline \multirow[t]{6}{*}{ información } & Familiares & 11 & 5,3 & 25 & 7,1 & 8 & 8,8 & \\
\hline & femeninas & & & & & & & \\
\hline & Colegio & 9 & 4,3 & 17 & 4,9 & 4 & 4,4 & \\
\hline & Amigas & 1 & 0,5 & 9 & 2,6 & 3 & 3,3 & \\
\hline & Padre & 12 & 5,7 & 14 & 4,0 & 2 & 2,2 & \\
\hline & Otro & 1 & 0,5 & 5 & 1,4 & 1 & 1,1 & \\
\hline
\end{tabular}

NS: diferencia no significativa.

mayoría de los casos las niñas no recordaron con exactitud la fecha en que esta ocurrió. Lograron precisar la edad en años asociándolo con el año escolar en que estaban y si fue invierno o verano, pero no el mes exacto, salvo en excepciones cuando coincidió con el cumpleaños, o alguna fiesta importante por lo tanto la edad a la menarquia en este estudio no se pudo registrar en meses sino en años cumplidos.

Si bien la mayoría de las niñas dijo que había recibido información previo a la menarquia, la información recibida en general era que la menstruación es un evento que solo ocurre en las mujeres, que la edad en que generalmente ocurre es entre los 12 y 13 años. Pudieron asociar la menstruación con crecimiento y un potencial logro para el embarazo. Pero tuvieron desconocimiento de hechos básicos acerca del ciclo menstrual, incluyendo cuando empieza y cuando termina, cuan frecuentemente ocurre y cuanto dura cada vez. Quizás el área de mayor familiaridad para ellas fueron las toallitas hi- giénicas. La mayoría de las niñas habían visto uno o más de estos artículos en el baño de la casa, en tiendas, en comerciales de televisión; pero también ocurrió que algunas de ellas, especialmente en las menores, que no entendieron con exactitud para que eran ocupados estos artículos.

Las fuentes de información sobre menstruación para las niñas fueron en primer lugar sus madres, luego el colegio, sus hermanas y amigas. Las madres fueron citadas como las fuentes mas apropiadas de asistencia menstrual; "cuando me vi toda manchada, llamé al tiro a mi mama al trabajo". En algunos casos fueron las propias madres que tomaron la iniciativa en conversar el tema, en otros casos fueron las niñas que iniciaron el tema. La información entregada por las madres se focalizó solo en aspectos higiénicos y biológicos ambiguos. Los mensajes fueron "ya eres una mujer, dejaste de ser niña y no puedes seguir haciendo cosas de niñas" (juegos), "tienes que cuidarte de los hombres, puedes quedar embarazada", pero sin explicar más 
Tabla III

DISTRIBUCIÓN DE VARIABLES SELECCIONADAS DE LAS ADOLESCENTES SEGÚN REACCIÓN A LA MENARQUIA

\begin{tabular}{|c|c|c|c|c|c|c|c|c|c|c|c|c|}
\hline \multirow[t]{2}{*}{ Variables } & \multirow[t]{2}{*}{ Categorías } & \multicolumn{2}{|c|}{ Mal } & \multicolumn{2}{|c|}{ Asustada } & \multicolumn{2}{|c|}{ Extraña } & \multicolumn{2}{|c|}{ Indiferente } & \multicolumn{2}{|c|}{ Feliz } & \multirow[t]{2}{*}{ Valor $p$} \\
\hline & & $\mathrm{n}$ & $\%$ & $\mathrm{n}$ & $\%$ & $\mathrm{n}$ & $\%$ & $\mathrm{n}$ & $\%$ & $\mathrm{n}$ & $\%$ & \\
\hline \multirow{2}{*}{$\begin{array}{l}\text { Relación con } \\
\text { padres }\end{array}$} & Buena & 95 & 53,1 & 85 & 52,8 & 74 & 47,1 & 9 & 29,0 & 124 & 53,0 & \multirow[t]{2}{*}{ NS } \\
\hline & No buena & 84 & 46,9 & 76 & 47,2 & 83 & 52,9 & 22 & 71,0 & 110 & 47,0 & \\
\hline \multirow{3}{*}{$\begin{array}{l}\text { Comunicación } \\
\text { familiar }\end{array}$} & Buena & 106 & 58,6 & 82 & 51,3 & 79 & 50,6 & 10 & 32,3 & 124 & 53,7 & \multirow[t]{3}{*}{ NS } \\
\hline & Regular & 26 & 14,4 & 32 & 20,0 & 33 & 21,2 & 11 & 35,5 & 34 & 14,7 & \\
\hline & Mala & 49 & 27,1 & 46 & 28,8 & 44 & 28,2 & 10 & 32,3 & 73 & 31,6 & \\
\hline Participación & $\mathrm{Si}$ & 86 & 49,1 & 70 & 43,8 & 75 & 48,4 & 12 & 38,7 & 112 & 49,1 & NS \\
\hline social & No & 89 & 50,9 & 90 & 56,3 & 80 & 51,6 & 19 & 61,3 & 116 & 50,9 & \\
\hline \multirow[t]{3}{*}{ Religión } & Católica & 103 & 57,5 & 100 & 62,1 & 110 & 71,0 & 18 & 58,1 & 135 & 57,9 & \multirow[t]{3}{*}{ NS } \\
\hline & Otra religión & 31 & 17,3 & 33 & 20,5 & 26 & 16,8 & 7 & 22,6 & 49 & 21,0 & \\
\hline & Sin religión & 45 & 25,1 & 28 & 17,4 & 19 & 12,3 & 6 & 19,4 & 49 & 21,0 & \\
\hline Antecedente & $\mathrm{Si}$ & 78 & 44,1 & 66 & 42,0 & 54 & 35,3 & 2 & 6,9 & 77 & 33,2 & 0,00 \\
\hline $\begin{array}{l}\text { de madre } \\
\text { adolescente }\end{array}$ & \multicolumn{11}{|c|}{ adolescente } & \\
\hline Con quien & Padres & 41 & 23,2 & 46 & 29,5 & 29 & 19,3 & 7 & 25,0 & 63 & 28,3 & NS \\
\hline conversa sobre & Familiares (fem) & 52 & 29,4 & 35 & 22,4 & 36 & 24,0 & 6 & 21,4 & 53 & 23,8 & \\
\hline \multirow[t]{3}{*}{ sexualidad } & Amigas & 69 & 39,0 & 49 & 31,4 & 62 & 41,3 & 13 & 46,4 & 79 & 35,4 & \\
\hline & Pololo & 11 & 6,2 & 22 & 14,1 & 18 & 12,0 & 1 & 3,6 & 25 & 11,2 & \\
\hline & Nadie & 4 & 2,3 & 4 & 2,6 & 5 & 3,3 & 1 & 3,6 & 3 & 1,3 & \\
\hline
\end{tabular}

NS: diferencia no significativa.

acerca de la biología de la menstruación, ciclo menstrual, órganos reproductivos. Por otro lado, si bien algunas niñas indicaron que habían conversado el tema con sus madres, ellas eran muy chicas cuando esta información se dio y no se acordaban muy bien de esta información cuando el evento ocurrió. Pocos padres participaron de este proceso educativo. Otras fuentes de información en este tema pero en baja frecuencia fueron películas $u$ otros materiales educativos en la escuela, amigas, doctora y otros adultos.

Las niñas que no habían recibido información acerca de la menstruación presentaron con mayor frecuencia reacciones de miedo y sorpresa especialmente las niñas que eran más pequeñas al momento de la menarquia. Ellas se asustaron mucho cuando se vieron manchadas y no sabían de donde provenía esta sangre. Asociaron el sangrado más bien a una herida.

Para la mayoría de las niñas algunas pistas iniciales sobre el tema fueron adquiridas a través de una variedad de fuentes informales, conversaciones en el baño escolar, comerciales de televisión, restos de sangre o toallitas vistas en baños públicos, observando a una hermana mayor o a la madre o descubriendo las toallitas en la casa.

La mayoría de las niñas indicaron que la menstruación es un tema difícil de confrontar abiertamente, que avergüenza. No es un tema que se converse con los muchachos, "cuando uno está con el periodo es un tema que se puede conversar con la madre, las hermanas, con las amigas", pero no es tema que 
se pueda conversar con el papá, con hermanos o amigos. Se considera un tema de mujer. Los muchachos pueden no entender o pueden molestar o bromear acerca de esto. Tampoco es un tema que se pueda conversar con cualquier mujer sino con aquellas que son más confiables.

En relación a reacciones emocionales a la primera menstruación estas abarcaron un amplio espectro que fue desde sentirse muy felices, contentas, sensación de sentirse más grandes, más maduras, hasta haberse sentido de lo peor, irritables, nerviosas, asustadas. Incluso presentaron molestias somáticas como sentirse mal, febril, débil, cansada. Las reacciones de las familias fueron en general poco estimuladoras. Muy pocas niñas manifestaron que sus familias reaccionaron felices, que las felicitaron, que les comunicaron a todos, que hicieron fiesta o que la mama la invito a comer afuera ese día. Los miedos asociados con la menstruación en la población estudiada se centraron en aquellas adolescentes que tuvieron su menarquia más temprana y/o no tuvieron adecuada información en relación al tema.

La menstruación altera las actividades normales de las niñas. Durante la menstruación según ellas, hay actividades que no pueden hacerse, como por ejemplo ir a la piscina o bañarse en la playa, estas son lejos las actividades más frecuentemente nombradas por las adolescentes como interrumpidas durante los días de la menstruación, porque "puede dar calambres" o "porque el flujo menstrual puede salir al agua". Otras actividades postergadas fueron hacer gimnasia o andar en bicicleta. Con frecuencia sintieron malestares físicos como jaquecas, dolores estomacales y otros que en algunos casos obligaron a algunas niñas ha permanecer en cama. Pocas consultaron médico por estas molestias porque sus propias madres, hermanas o abuelas habían tenido los mismos malestares y asumían esto como natural. Las niñas sienten que están limitadas en varios aspectos, por ejemplo, en esos días no podrían usar ropa de color blanco porque sería más notorio en caso que el flujo menstrual manchara la ropa y se notara.

La mayoría de las entrevistadas creen que antes que llegue la regla a las niñas, una de las principales preocupaciones que presentan es no estar usando las toallitas al momento de la menstruación y manchen la ropa. Peor todavía si esta circunstancia ocurre en el colegio, sobre todo en colegios mixtos o con profesores varones. Otra preocupación era tener reglas muy abundantes y/o que durará varios días.

En general, los miedos asociados con la menstruación en la población estudiada se centraron en aquellas adolescentes que tuvieron su menarquia más temprana y en aquellas que no tuvieron adecuada información al inicio de la menstruación.

\section{CONCLUSIÓN}

Los hallazgos de este estudio permiten conocer la precariedad de la información en sexualidad y en especial sobre la menstruación con que llegan las niñas a enfrentar y vivenciar su menarquia, lo que sugiere la necesidad de que profesionales de la salud y educación bien calificados preparen mejor a las madres, a las familias y educadores en esta temática.

\section{BIBLIOGRAFÍA}

1. Simmons RG, Blyth DA, Van Cleave EF, Bush DM. Entry into early adolescence: the impact of school structure, puberty and early dating on self-esteem. Am Sociol Rev 1979;44(6):948-67.

2. Smith EA, Uldry JR, Morris NM. Pubertal development and friens: a biosocial explanation of adolescent sexual behavior. J Health Soc Behav 1985;26(3):18392.

3. de La Rochebrochard E. Age at puberty of girls and boys in France: Measurements from a survey on adolescent sexuality. Population: An English Selection 2000;12:51-79.

4. Garg S, Sharma N, Sahay R. Socio-cultural aspects of menstruation in a urban slum in Delhi, India. Reprod Health Matters 2001;9(17):16-25.

5. Muscari ME, Faherty J, Catalino C. Little Women: early menarche in rural girls. Pediatr Nurs 1998;24(1):115.

6. Anson O. Exploring the bio-psycho-social approach to premenstrual experiences. Soc Sci Med 1999;49(1):67-80.

7. McGrory A. Menarche: Responses of early adolescent females, Adolescence 1990;25(98):265-70.

8. Benjet C, Hernandez-Guzman L. Gender differences in psychological well-being of Mexican early adolescents, Adolescence 2001;36(141):47-65.

9. Ruble DN, Brooks-Gunn J. The experience of menarche. Child Devel 1982;53(6):1557-66.

10. Brooks-Gunn J, Ruble DN. The development of menstrual-related beliefs and behaviors during early adolescence. Child Devel 1982;53(6):1567-77.

11. Chaturverdi SK, Chandra PS. Sociocultural aspects of menstrual attitudes and premenstrual experiences in India. Soc Sci Med 1991;32(3):349-51.

12. Clarke AE, Ruble DN. Young Adolescents' beliefs concerning menstruation. Child Devel 1978;49(1):231-4.

13. Yeung DYL, So-kum Tang C, Lee A. Psychosocial and cultural factors influencing expectations of menarche: a study on chinese premenarcheal teenage girls. J Adol Research 2005,20(1):118-35.

14. Brooks J, Ruble D, Clarke A. College women's attitudes and expectations concerning menstrual-related changes. Psychosom Med 1977;39(5):288-98. 
15. Ruble DN. Premenstrual symptoms: reinterpretation. Science 1977;197(4300):291-2.

16. Wilcoxon LH, Schrader SL, Sherif CW. Daily self-reports on activities, life events, moods, and somatic changes during the menstrual cycle. Psychosom Medicine 1976, 33:399-417.

17. Koff E, Rierdan J. Premenarcheal expectations and post-menarcheal experiences of positive and negative menstrual related changes. J Adolesc Health 1996;18(4):286-91.

18. Marvan ML, Vacio A, Espinosa-Hernandez G. A comparison of menstrual changes expected by pre-menarcheal adolescents and changes actually experienced by post-menarcheal adolescents in Mexico. J Sch Health 2001;71(9):458-61.

19. Koff E, Rierdan J, Sheingold K. Memories of menar- che: age, preparation and prior knowledge as determinants of initial menstrual experience. J Youth Adolesc 1982:11(1):1-9.

20. Morse JM, Doan HM. Adolescents' response to menarche. J Sch Health 1987;57(9):385-9.

21. Graber JA, Brooks-Gunn J, Warren MP. The antecedents of menarcheal age: heredity, family environment, and stressful life events. Child Dev 1995, 66(2):346-59.

22. Swenson I, Havens B. Menarche and menstruation: a review of the literature. J Community Health Nurs 1987;4(4):199-210.

23. Greif EB, Ulman KJ. The psychological impact of menarche on early adolescent females: a review of the literature. Child Dev 1982;53(6):1413-30. 